\title{
Users' Guide to the JABFP Online Manuscript Management System
}

\author{
Anne Victoria Neale, PhD, MPH, and Nancy Jacobson
}

Early in 2003, the Fournal of the American Board of Family Practice adopted Rapid Review, an on-line program for manuscript submission, peer review, and tracking. We no longer accept hard copy submissions for publication consideration, unless special arrangements have been made.

Rapid Review is a product of Cadmus KnowledgeWorks, which handles the $\mathcal{F} A B F P$ publication production process. Rapid Review is a comprehensive database that has many beneficial features for authors, peer reviewers, and editors. There is also greater efficiency in the editorial office without the need to receive paper submissions and in turn send these out to peer reviewers, who then mail or fax their reviews back in. All communication is via email, and all correspondence, reviews, and submissions are stored electronically.

Authors no longer need to print and mail hard copies and electronic files on disk (except for figures, in some cases; see below). They upload their manuscripts through the web portal on the Journal web site (http://www.jabfp.org). Authors can also track the progress of their manuscripts through the peer review system. All correspondence to authors is sent via email messages that originate in the Rapid Review system.

Peer reviewers receive a request to review a manuscript through an automated Rapid Review e-mail message. After "accepting" the assignment, reviewers can download the manuscript. They fill out an objective rating form and also provide a written critique of the paper. Reviewers will find a portal separate from that for authors on the Journal web site (http://www.jabfp.org).

Editors access the peer reviewers' critique and recommendation and write a decision letter to au-

Submitted, revised, 22 December 2004.

From the $7 A B F P$. Address correspondence to Dr. Anne Victoria Neale, Department of Family Medicine, 101 E. Alexandrine, Wayne State University, Detroit, MI 48201 (e-mail: jabfp@med.wayne.edu). thors, all within the Rapid Review system. Authors who are invited to revise and resubmit their papers then upload the new version into the system. The history of each submission and all correspondence is retained within the system.

Rapid Review also contains a database of reviewers, which is used to match reviewer expertise with the content and methodology of each manuscript. Peer reviewers are asked to develop their own profiles with contact information and areas of expertise.

The following is based on questions we have received from users, both peer reviewers and authors. Users should first read the detailed instructions for submitting a manuscript via Rapid Review that are provided in the Information for Authors section on the journal home page (http://www.jabfp. org). Below are some additional trouble-shooting tips, developed in response to frequently asked questions.

\section{Logging in to Rapid Review}

Rapid Review has two different gateways. One is for authors, and the other is for editors, reviewers, and staff. Both of these portals can be reached by going to www.jabfp.org and clicking on the appropriate link. Authors who also have reviewer status will need to be sure that they are logging in to the correct link for the duties that they wish to perform.

\section{Loading a Manuscript}

When loading a manuscript, Rapid Review will send the author a series of emails. The first may state that the manuscript submission is not complete; this is often followed by another email asking that the author $\log$ on and check the PDF file to make sure it loaded correctly. The email stating that the submission is incomplete can be ignored. Authors should check the conversion of their PDF, and if it is acceptable, finish the submission process by clicking that the PDF meets with their approval. 
Authors must be sure to include in the submitted .DOC file all information that they want published. This includes the abstract, acknowledgments, conflict of interest statements, funding sources, and the complete author list. Although the Rapid Review system asks for this information as part of the manuscript submission process, this information is for the editors only; the copy editor will receive only information that is included in the manuscript .DOC file. The only items that should not appear in the .DOC file are tables (which should be in a separate .DOC file) and figures (which should be in separate .TIF or .EPS files).

\section{Submitting Figures and Tables}

We strongly encourage authors to submit figures and tables as separate digital files. This will speed the production process if the manuscript is accepted. Otherwise, authors will need to provide high-quality hard copies by regular mail. Please note that JPEG files are not suitable for printing. For more information about how to prepare digital art for printing, please visit http://www.cadmusknowledgeworks.com/da. For tables, please use Microsoft Word's table feature. No other graphics features of Word should be used when creating the tables (text boxes, graphics boxes, drawing, etc). Cells can be copied from an Excel spreadsheet and pasted into a Word document, but Excel files should not be embedded as objects.

\section{Accepting or Declining a Review}

Reviewers receive an email generated by Rapid Review notifying them that they have been selected by the editorial staff to review a manuscript. The reviewer must click on the link in this message to "Accept" or "Decline" the review. Sending an email reply to the manuscript staff will NOT activate the reviewer's decision in the system. This may result in a reviewer who has declined via email to still receive reminders from the system. Rapid Review will only log the decision when the reviewer clicks on the link, goes to the appropriate page, and clicks "Accept" or "Decline."

\section{"Due By" Dates}

There is some confusion on the dates that Rapid Review generates stating that a decision to review or an actual review is due by a certain date. Even if it is past the date, the assignment can still be accepted. When reviewers need a longer period, they can contact the manuscript staff and provide a date of expected completion. The Rapid Review program may still send a tardy review notice, but if reviewers have made arrangements with the office, they can disregard the notice. We cannot modify these 'due by' dates that are system-generated, so please be patient with Rapid Review e-mail reminders.

\section{Pop-Up Access for PDFs}

Rapid Review is a pop-up dependent program. That means that your computer has to be set to allow pop-ups to be able to take advantage of the full functions that Rapid Review has to offer. When the upgrades for the Microsoft XP (Service Pack 2) went into effect, they further disabled pop-ups.

If you are trying to view a PDF of a manuscript and experience a pop-up block:

- Click the PDF icon.

- You will hear a beep, and a tan bar will appear at the top of a new screen.

- The tan bar will read "To help protect your security, Internet Explorer blocked this site from downloading files to your computer. Click here for options."

- Click the tan bar.

- Select "Download files."

- DO NOT close the window; minimize it.

- This will return you to the manuscript page with the PDF icon.

- Click PDF icon again.

- Window will appear offering you to "open" or "save" the file.

- You may now view the file.

\section{Miscellaneous Tips}

- You must click on a prompt such as "GO" or "Continue" to move forward; the enter key will not work.

- Save your work! Be sure to frequently save your work when submitting a peer review or when creating an author or reviewer account. If there is no activity after a couple of minutes, your Rapid Review session will end, and any unsaved work will be lost. If you can't finish your work in one session, save it and come back to finish later. 